\title{
ARTICLE \\ Hydroelastic Behaviour and Analysis of Marine Structures
}

\section{Fuat Kara*}

Sheffield Hallam University, Howard Street, Sheffield, S1, 1WB, UK

\section{ARTICLE INFO}

Article history

Received: 7 December 2020

Accepted: 11 January 2021

Published Online: 31 January 2021

\section{Keywords:}

Hydroelasticity

Boundary integral equation method

Transient wave Green function

Time domain

Neumman-Kelvin linearisation

Euler-Bernoulli beam

\section{Introduction}

The fluid-structure interactions of the floating system result in rigid or elastic motions which depend on the disturbance of pressure field around marine structures. If the effects of the rigid body modes are greater than elastic modes, the contribution of elastic modes to the disturbance of the pressure field is not important which implicitly means that the floating system is a stiff structure and the structural and hydrodynamic analyses are weakly coupled and two analyses can be done separately ${ }^{[1-3]}$. If the radiation field is affected significantly with the structural deformation, the hydroelastic analysis, which considers the fully coupling of the structural and hydrodynamic analysis, needs to be taken into account for the prediction of the motion of the floating systems ${ }^{[4-5]}$.

\section{ABSTRACT}

Hydroelasticity of marine structures with and without forward speed is studied directly using time dependent Boundary Integral Equation Method with Neumann-Kelvin linearisation where the potential is considered as the impulsive velocity potential. The exciting and radiation hydrodynamic parameters are predicted in time with transient wave Green function whilst the structural analysis is solved with Euler-Bernoulli beam method at which modeshapes are defined analytically. The modal analysis is used to approximate the hydroelastic behaviour of the floating systems through fully coupling of the structural and hydrodynamic analyses. As it is expected, it is found with numerical experience that the effects of the rigid body modes are greater than elastic modes in the case of stiff structures. The predicted numerical results of the present in-house computational tool ITU-WAVE are compared with experimental results for validation purposes and show the acceptable agreements.

Hydroelasticity plays significant role especially for the high-speed crafts and larger marine vehicles. In the case of larger floating systems, the incident wave frequencies and natural frequency are closer to each other whilst the frequency range that results in the hull-girder vibrations approaches the encounter frequency of the high-speed crafts.

The elastic deflection of the structures in air can be predicted accurately with modeshapes, however, in the case of floating systems, due to the effects of the radiation pressure field the modeshapes need to be determined at current time and the elastic deformation of the floating structures may be predicted with the modeshape superpositions. The dry modeshapes in air can be used to predict the elastic deformation of the floating systems ${ }^{[1]}$, alternatively, elastic deflection in fluid may be represented with orthogonal

*Corresponding Author:

Fuat Kara,

Howard Street, Sheffield, S1, 1WB, UK;

E-mail: fuat.kara@shu.ac.uk 
polynomials ${ }^{[4]}$ or orthogonal beam of a uniform beam ${ }^{[6]}$. The rigid body modes are considered as the part of the elastic modes under the same boundary conditions in the hydroelastic analysis. It is expected that the effects of higher modes on elastic motions would not be significant as the interactions between the natural frequencies of the higher modes and incident wave frequencies would be weaker compared to those of lower modes.

The frequency domain ${ }^{[1]}$ or time domain ${ }^{[2-3,5]}$ methods may be used to predict the hydroelastic behaviour and analysis of the floating systems. Two-dimensional strip theory methods ${ }^{[7-8]}$ are one of the options that may be used for the approximations of the hydrodynamic and hydroelastic parameters. However, the strip theory method has shortcomings at bow and stern regions of floating systems, global loads approximations, complex geometries, high forward speed, and low frequency ranges. These shortcomings of the strip theory methods could be overcome with the use of three-dimensional methods both in frequency and time domains which include the interactions amongst panels automatically.

The hydrodynamic parameters can be predicted with two popular numerical methods both in frequency and time domains including wave Green function ${ }^{[2,9-10]}$ and Rankine panel methods ${ }^{[11-12]}$. The wave Green function methods satisfy the condition at infinity and free surface boundary condition automatically which results in only discretisation of the body surface under mean water level to satisfy the body boundary condition. In the case of Rankine panel method, both some part of the free surface and body surface under mean position need to be discretised to satisfy the condition at infinity and body boundary condition respectively. in the context of linear analysis, the frequency and time domain results depend on each other via Fourier transform. The time domain methods are better suited to predict the nonlinear cable forces, unsteady manoeuvring, motions with large amplitudes, and nonconstant forward speed compared to frequency domain methods.

The finite element method with three-dimensional shell elements ${ }^{[12]}$, two-dimensional plate elements using Kirchoff or Mindlin plates ${ }^{[2-3]}$ or one-dimensional beam elements using Vlasov, Timoshenko and Euler-Bernoulli beams ${ }^{[1,5,13]}$ are used to predict the hydroelastic behaviour of the floating systems including eigenvectors and eigenvalues. The body boundary conditions for elastic floating bodies, which include rigid body boundary conditions, are obtained ${ }^{[1,4-5]}$ through the extension of unified theory ${ }^{[14]}$.

The fluid forces and velocities are predicted with potential three-dimensional formulation and transient wave Green function whilst the structural parameters are obtained with one-dimensional Euler-Bernoulli beam approximation in the present paper. The free stream is selected as a basis flow which results in the linearisation of the initial boundary value problem. The time dependent boundary integral equation is obtained using the three-dimensional transient wave Green function and Green theorem ${ }^{[2-3,5,15-21]}$. The present in-house ITU-WAVE computational numerical results are validated against the experimental results ${ }^{[22-23]}$ which shows acceptable agreements.

\section{Equation of Motion of Elastic Floating Sys- tems}

The body-fixed Cartesian coordinate system $\vec{x}=(x, y, z)$ in Fig. 1 is used to represent the fluid behaviour around the elastic floating systems. The positive directions of $\mathrm{x}, \mathrm{y}$ and $\mathrm{z}$ directions are defined with the positive direction of a right-hand coordinate system. The free surface is coincident with $\mathrm{z}=0$ plane. The elastic floating body moves forward in positive $\mathrm{x}$ direction with forward speed $U_{0}$. The fluid domain in Fig. 1 is identified with boundary at infinity $S_{\infty}$, boundary on free surface , $S_{f}(t)$ boundary of body surface $S b(t)$ and interaction between free surface and body $(t)^{[2,15]}$.

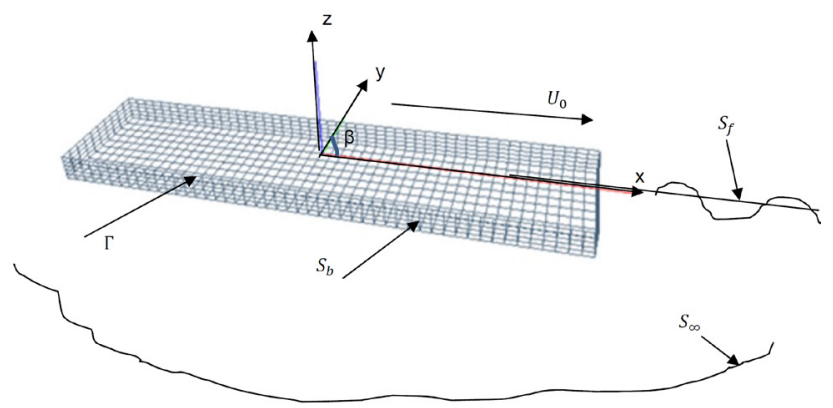

Figure 1. Fluid boundaries and Coordinate system

It is assumed that the fluid is inviscid, incompressible and the flow of the fluid is irrotational. These assumptions result in the fluid velocities to be represented as the gradient of the velocity potential $\vec{V}(\vec{x}, t)=\nabla \Phi(\vec{x}, t)$ and Laplace equation governs the fluid domain $\nabla^{2} \Phi(\vec{x}, t)=0$. The time dependent equation of motions includes the inertia term, elastic hydrostatic and hydrodynamic restoring coefficients which is represented with convolution integral and excitation force due to incident wave which is also represented with convolution integral ${ }^{[24]}$.

$\sum_{k=1}^{K}\left(M_{j k}+a_{j k}\right) \ddot{x}_{k}(t)+b_{j k} \dot{x}_{k}(t)+$ 


$$
\begin{aligned}
& \left(k_{j k}+C_{j k}+c_{j k}\right) x_{k}(t)+\int_{0}^{t} d \tau K_{j k}(t-\tau) \dot{x}_{k}(\tau) \\
& =\int_{-\infty}^{\infty} d \tau K_{j D}(t-\tau) \zeta(\tau)
\end{aligned}
$$

Where $\mathrm{j}=1,2,3, \ldots, \mathrm{K}$ and the coefficients in Eq. (1) are given as

$$
\begin{aligned}
M_{j k} & =\int_{-L / 2}^{L / 2} m \omega_{j}(x) \omega_{k}(x) d x \\
k_{j k} & =\int_{-L / 2}^{L / 2} E I \omega_{j}^{\prime \prime}(x) \omega_{k}^{\prime \prime}(x) d x \\
C_{j k} & =\rho g \iint_{S_{0}} d S_{Q}\left(\omega_{j}+z \nabla \cdot \vec{u}_{j}\right) n_{k}
\end{aligned}
$$

The displacement, velocity and acceleration of elastic floating system are given in Eq. (1) with $x_{k}(t), \dot{x}_{k}(t)$ and $\ddot{x}_{k}(t)$ respectively. The structural mass matrix Eq. (2) where $\omega_{j}$ is the part of the modeshapes $\vec{u}_{j}=\left(0,0, \omega_{j}\right)$, structural stiffness matrix Eq. (3), and hydrostatic restoring force coefficients Eq. (4) are represented with $M_{j k}$, $k_{j k}$, and $C_{j k}$ respectively. The nondimensional stiffness parameter $S=E I / \rho g L^{5}$, which is the function of the hydrostatic restoring force and structural stiffness, is developed assuming the mass $m$ per unit length and structural stiffness $E I$ are uniformly distributed along longitudinal direction $^{[25]}$. The nondimensional parameter $S=0$ is used for fully elastic structure whilst $S=\infty$ is for fully rigid structures. The generalised modes with separation of variables are used to represent the total displacements of the elastic floating structures.

$\vec{u}(x, y, z, t)=\sum_{k=1}^{K} \vec{u}_{k}(x, y, z) x_{k}(t)$

Where time dependent principal coordinates $x_{k}(t)$ and modeshapes depending on spatial variables $(x, y$, $z)$ are given with $x_{k}(t)$ and $\vec{u}_{k}(x, y, z)$ respectively. The elastic modeshapes $\vec{u}_{k}(x, y, z)$ include the rigid body modes such that $\vec{u}_{3}(0,0,1)$ is used for heave mode whilst $\vec{u}_{5}(0,1,0) \times \vec{x}$ is used for pitch mode.

$$
\begin{aligned}
& a_{j k}=\rho \iint_{S_{0}} d S_{Q} \Psi_{1 k} n_{j} \\
& b_{j k}=\rho \iint_{S_{0}} d S_{Q}\left(\Psi_{1 k} m_{j}-\Psi_{2 k} n_{j}\right) \\
& c_{j k}=-\rho \iint_{S_{0}} d S_{Q} \Psi_{2 k} m_{j}
\end{aligned}
$$

$K_{j k}(t)=\rho \iint_{S_{0}} d S_{Q}\left\{\frac{\partial}{\partial t} \chi_{k}(t) n_{j}-\chi_{k}(t) m_{j}\right\}$

The convolution integral at the left-hand side of Eq. (1) is the function of the radiation Impulse Response Functions (IRFs) $K j k(t)$ and the velocity $\dot{x}_{k}(t)$ of the elastic floating systems. This convolution integral represents the wave damping or hydrodynamic restoring force coefficients. The time dependent IRFs $K j k(t)$ represent the memory or free surface effect. The frequency and time independent infinite added mass, damping and restoring force related coefficients are given with $a_{k j}, b_{j k}$, and $c_{j k}$ respectively. The time independent instantaneous fluid response and the steady displacement of the elastic structure due to forward speed effect are given with $\psi_{1 k}$ and $\psi_{2 k}$ respectively. The transient behaviour of elastic floating system due to free surface effect is considered with the time dependent potential $x k(t)^{[26]}$.

$$
\begin{aligned}
& F_{j D}(t)=\int_{-\infty}^{\infty} d \tau K_{j D}(t-\tau) \zeta(\tau)=\int_{-\infty}^{\infty} d \tau\left\{K_{j S}(t-\tau)\right. \\
& \left.+K_{j I}(t-\tau)\right\} \zeta(\tau) \\
& K_{j I}(t)=\iint_{S_{0}} d S_{Q} \hat{p}(t) n_{j} \\
& K_{j S}(t)=\rho \iint_{S_{0}} d S_{Q}\left\{-\frac{\partial}{\partial t} \widehat{\phi}_{S}(t) n_{j}+\widehat{\phi}_{S}(t) m_{j}\right\}
\end{aligned}
$$

The convolution integral in the right-hand side of Eq. (1) is the function of the exciting IRFs $K_{j D}(t)$ and incident wave elevation $\zeta(t)$ which is impulsive and defined at the centre of the fixed coordinate system with heading angle $\beta$ in Fig. 1. The exciting IRFs $K_{j D}(t)$ have two components; one of them is due to incident waves $K_{j l}(t)$ in Eq. (11) whilst the second one is due to the effects of the scattering waves $K_{j s}(t)$ in Eq. (12) respectively. The time dependent impulsive pressure is given with $\hat{p}(t)$ whilst the scattering wave potential which results from the interactions of the incident waves with floating systems is given with $\widehat{\phi}_{S}(t)^{[10]}$.

\section{Deflection of Elastic Floating Bodies}

The bending moments and shear forces of elastic floating systems with three-dimensional finite element methods ${ }^{[2-3,27]}$ can be predicted with the free vibration of the marine structures defining the deformations with modeshapes. Free-free beam ${ }^{[6]}$, Chebyshev and Legendre polynomials or Fourier series ${ }^{[4]}$ could be also used to determine the deformation of the elastic marine structures. Euler-Bernoulli beam theory with free-free beam modes and nondimensional coordinates $q=2 x / L$, which satisfy the boundary conditions of zero shear forces and 
bending moments at the end points, is used in the present paper $^{[6]}$.

$u_{2 j}(q)=\frac{1}{2}\left(\frac{\cos \left(\kappa_{2 j} q\right)}{\cos \left(\kappa_{2 j}\right)}+\frac{\cosh \left(\kappa_{2 j} q\right)}{\cosh \left(\kappa_{2 j}\right)}\right)$

$u_{2 j+1}(q)=\frac{1}{2}\left(\frac{\sin \left(\kappa_{2 j+1} q\right)}{\sin \left(\kappa_{2 j+1}\right)}+\frac{\sinh \left(\kappa_{2 j+1} q\right)}{\sinh \left(\kappa_{2 j+1}\right)}\right)$

Where nondimensional normalized coordinate $q \in[-1,1]$ and mode index $\mathrm{j}=1,2,3, \ldots$. As the rigid body modes are the part of the elastic modes, the first elastic mode is the heave rigid body mode $u_{0}(q)=1$ whilst the second elastic mode is the pitch rigid body mode $u_{1}(q)=-q L / 2$. The $\vec{u}_{k}=\left(0,0, u_{k}(q)\right)$ vector represents the modeshapes in Eq. (13) and Eq. (14) in which the $k_{j}$ parameters are determined with Eq. (15).

$(-1)^{j} \kappa_{j} \tan _{j}+\tanh \kappa_{j}=0$

The free-free beam modeshapes of the elastic modes, which include the rigid modes of heave and pitch, are presented in Fig. 2.

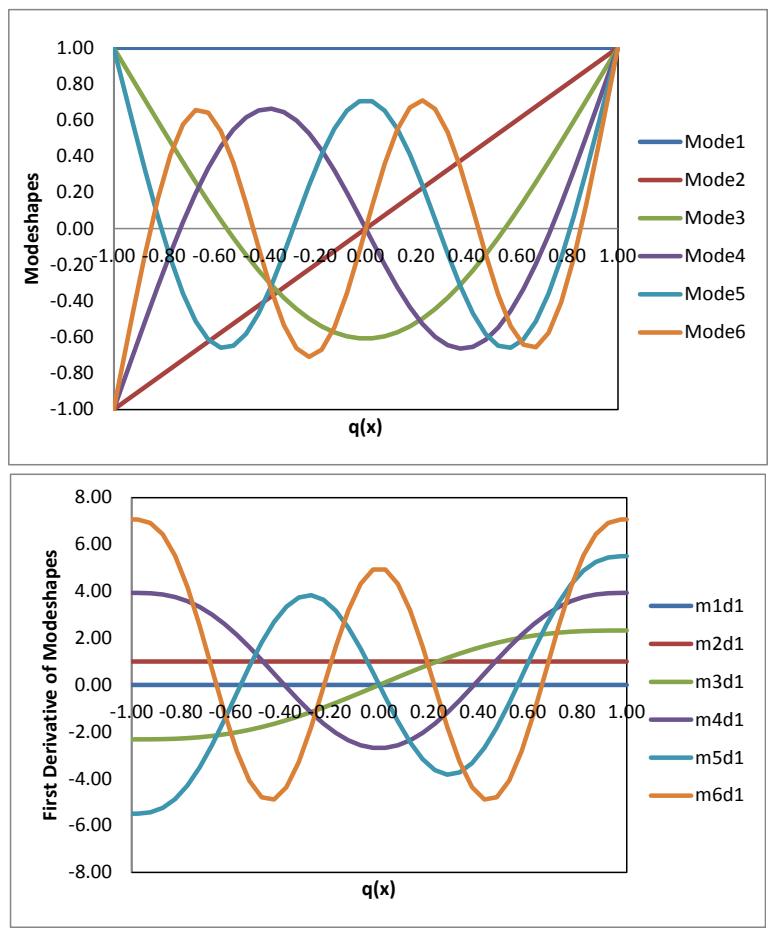

Figure 2. The first six free-free beam modes and the first six first derivatives of free-free beam modes which in-

clude the rigid body modes of heave and pitch

\subsection{An Elastic Slender Barge for Hydroelastic Analysis}

An elastic slender barge in head seas floating freely is used to predict the deformable modes which include heave and pitch rigid body modes. The elastic barge has zero speed with draft to beam ratio $\mathrm{T} / \mathrm{B}=0.5$ and length to beam ratio $\mathrm{L} / \mathrm{B}=10$. Total 516 panels are used to discretise the elastic barge including 3 panel in vertical direction, 6 panels in transverse direction and 40 panels in longitudinal direction as presented in Fig. 3.

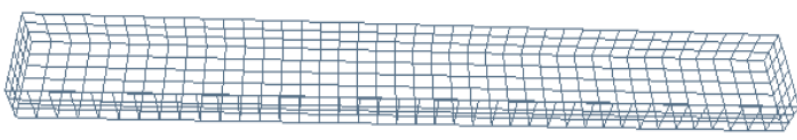

Figure 3. Total 516 panels are used with 3 panel in vertical direction, 6 panels in transverse direction and 40 panels in longitudinal direction

It is assumed that the structural stiffness and mass are distributed uniformly in longitudinal direction of the elastic floating systems such that mass matrix with constant mass distribution ${ }^{[4]}$ is given as

$M_{j k}=\int_{-L / 2}^{L / 2} m \omega_{j}(2 x / L) \omega_{k}(2 x / L) d x=\frac{M}{4} \delta_{j k}$

where the Kroenecker delta function and elastic beam's total mass are given with $\delta_{j k}$ and $\mathrm{M}=\mathrm{mL}$ respectively. The structural stiffness $k_{j k}$ with constant $\mathrm{EI}$ is given as

$k_{j k}=\int_{-L / 2}^{L / 2} E I \omega_{j}^{\prime \prime}(2 x / L) \omega_{k}{ }^{\prime \prime}(2 x / L) d x=4 \frac{E I}{L^{3}} \kappa_{j}^{4} \delta_{j k}$

where the modulus of elasticity and second moment of inertial are given with $\mathrm{E}$ and I respectively. The off-diagonal elements of the stiffness and mass matrices are zero as these matrices are symmetric.

The deformable nondimensional radiation IRFs of elastic barge including $7,8,9, \ldots, 14$ elastic modes are presented in Fig. 4 in which the area under each elastic mode represents the available energy for each mode. It may be noticed in Fig. 4 that available energy decreases with increasing elastic mode numbers which implies that the effects of the higher elastic modes to the total displacements are not significant.

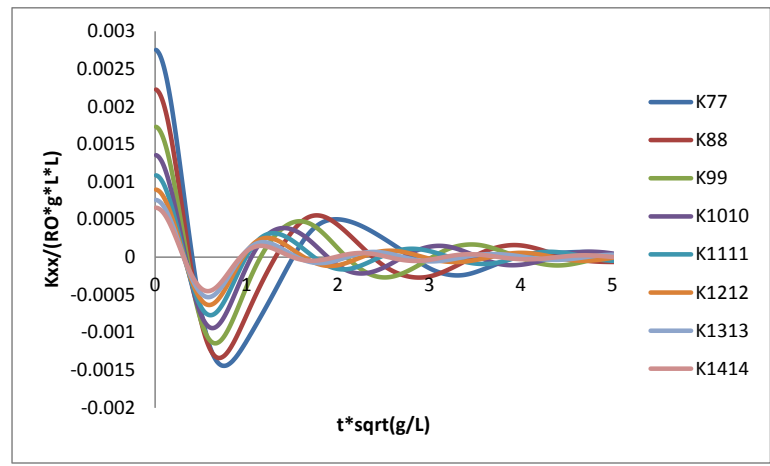

Figure 4. The first 8 deformable modes of nondimensional radiation IRFs of the elastic barge 
The time domain radiation IRFs $K_{j k}(t)$ and frequency domain added-mass $A_{j k}(\omega)$ and damping coefficients $B_{j k}(\omega)$ in the context of linear analysis depend on each other through Fourier transform for each elastic mode as presented in Eq. (18) and (19).

$$
\begin{aligned}
& A_{j k}(\omega)=a_{j k}-\frac{1}{\omega} \int_{0}^{t} d \tau K_{j k}(\tau) \sin (\omega \tau)-\frac{c_{j k}}{\omega^{2}} \\
& B_{j k}(\omega)=b_{j k}+\int_{0}^{t} d \tau K_{j k}(\tau) \cos (\omega \tau)
\end{aligned}
$$

Fourier transform of radiation IRFs $K_{j k}(t)$ in Fig. 4 is used to obtain the frequency dependent added-mass $A_{j k}(\omega)$ and damping coefficients $B_{j k}(\omega)$ in Fig. 5. As in the case of IRFs in Fig. 4, the same behaviour may be observed in Fig. 5 as the amplitude of the added-mass and damping coefficients are decreasing with the increasing mode numbers.

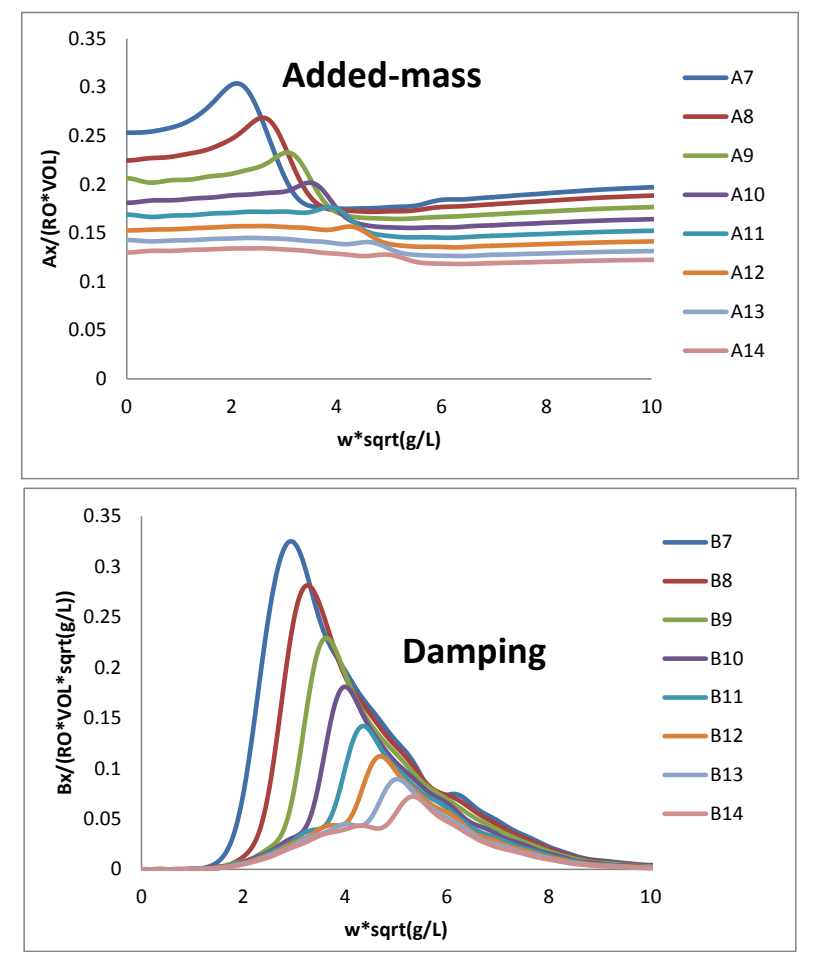

Figure 5. The first 8 deformable modes of nondimensional added-mass and damping coefficients
The regular or irregular incident wave $\zeta(t)$, which is defined at the centre of body-fixed coordinate system in Fig. 1, is used to excite the elastic floating systems whilst the time dependent incident wave potential $\varphi_{I}(\vec{x}, t)$ is known and analytically defined in Eq. 20.

$\varphi_{I}(\vec{x}, t)=\frac{i g}{\omega} e^{k(z-i \varpi)} e^{i \omega_{e} t}$

Where the absolute frequency of the incident waves, encounter frequency, wave number in infinite water depth, incident wave heading angle and positions on the free surface with respect to heading angle are given with $\omega$,

$\omega_{e}=\omega-U_{0} k \cos (\beta), k=\omega^{2} / g, \beta$ and $\varpi=$ $x \cos (\beta)+y \sin (\beta)$ respectively.

Froude-Krylov IRFs, diffraction IRFs and exciting IRFs which are obtained by the superposition of Froude-Krylov and diffraction IRFs are presented in Fig.6 for the first three deformable modes including 7, 8 and 9 modes. The area under these IRFs represent the available energy that would be absorbed by the elastic floating systems during the interactions of incident waves and elastic marine structures.

As in the case of radiation analysis, the time dependent Froude-Krylov, diffraction and exciting IRFs are linked to frequency dependent Froude-Krylov, diffraction and exciting force amplitude and phase angles through Fourier transform as presented in Eq. (21).

$X_{j}\left(\omega_{e}\right)=\int_{-\infty}^{\infty} d \tau\left[K_{j l}(\tau)+K_{j S}(\tau)\right] e^{-i \omega \tau}$

where the frequency dependent exciting force parameters in complex form are given with $X_{j}\left(\omega_{e}\right)$ in which the exciting force amplitudes including Froude-Krylov and diffraction are the absolute value of complex exciting force $X_{j}\left(\omega_{e}\right)$ whilst those of phase angles is the arguments of $X_{j}\left(\omega_{e}\right)$. The frequency dependent exciting force amplitudes and phase angles in Fig. 7 are obtained via Fourier transform of time dependent IRFs in Fig. 6.

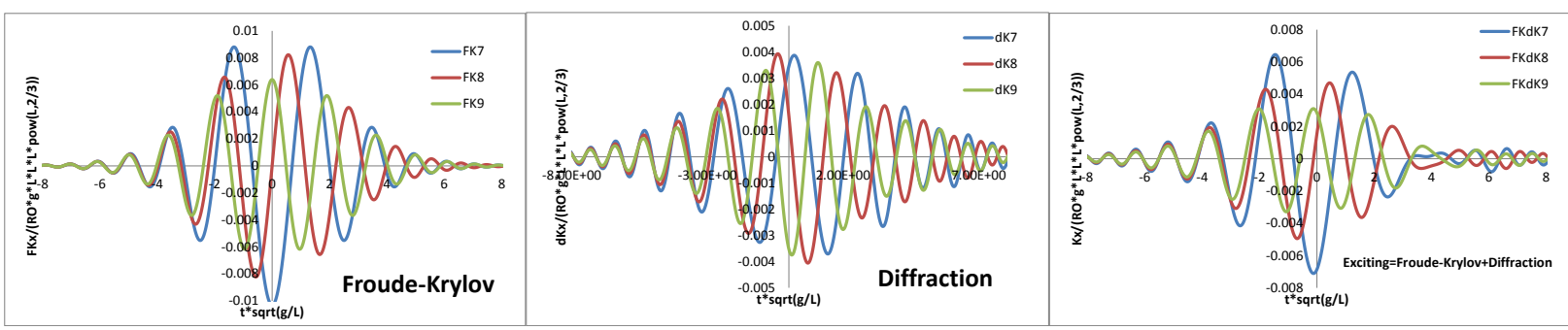

Figure 6. Nondimensional Froude-Krylov, diffraction, and exciting IRFs for the first three deformable modes of 7, 8 and 9 


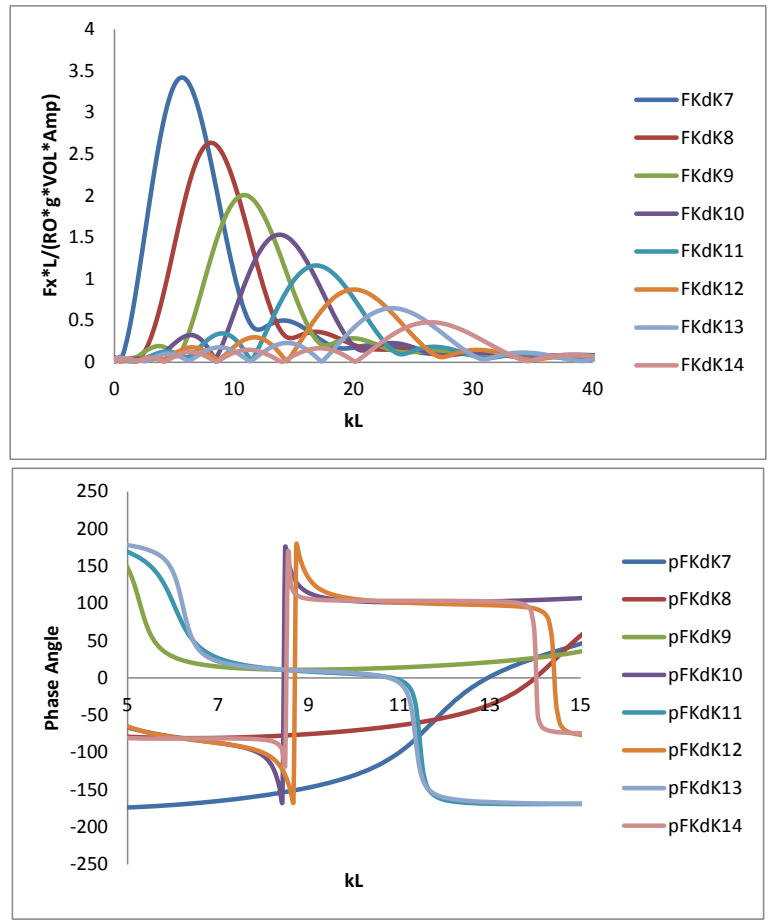

Figure 7. Nondimensional exciting force amplitudes and phase angles of elastic slender barge for the first 8 deformable modes including 7, 8 and 9 modes

Response Amplitude Operators (RAOs) or deflections of an elastic floating systems in frequency domain may be written as in Eq. (22).

$x_{k}(q)=\sum_{k=1}^{K} x_{k}(\omega) u_{k}(q)$

Where normalised coordinate in nondimensional form is given with $q=2 x / L$. The $x_{k}(q)$, which is the function of the absolute wave frequency $\omega$ and normalised coordinates $q$, is the complex form of the elastic RAOs in which the absolute value is the amplitude of the response whilst the argument is the phase angle of the response. The nondimensional stiffness parameter $\mathrm{S}$ for fully rigid, hydroelastic effect significant, and fully flexible elastic slender barge are given with $S=1, S=10^{-5}$, and $S=0$ for the first eight deformable elastic modes in Fig. 8.

The effects of the deformable modes to the response of elastic slender barge in the case of fully rigid condition $\mathrm{S}=1$ is not significant and almost zero whilst in the case of fully elastic condition $S=0$, the effects of elastic deformable modes to RAOs are considerable and significant.

The RAOs, which are predicted using Eq. (22), at stern $(-\mathrm{L} / 2)$, midship $(0 \times \mathrm{L})$ and bow $(\mathrm{L} / 2)$, are presented for different stiffness factor S. It may be noticed from Fig. 9 that there are no differences with the results of $S=10^{-1}$ and rigid body motion whilst there are also no differences with those of stiffness parameter $\mathrm{S}=10^{-8}$ and $\mathrm{S}=0$. It can be withdrawn from Fig. 9 results that the range of $10^{-8}<\mathrm{S}<$ $10^{-2}$ has the significant hydroelastic effects.

It is known that the maximum response occurs when the elastic floating body length equals the incident wavelength $(\omega=0.785 \mathrm{rad} / \mathrm{s})$. The RAOs and the superposition of RAOs are presented in Table 1 using eight deformable elastic modes in the case of nondimensional stiffness factor $\mathrm{S}=10^{\wedge}-4$ at the location of $\mathrm{L} / 4$ and $\mathrm{L} / 2$ (bow). The convergence up to three decimals are achieved with four elastic deformable modes as can be observed from Table 1.

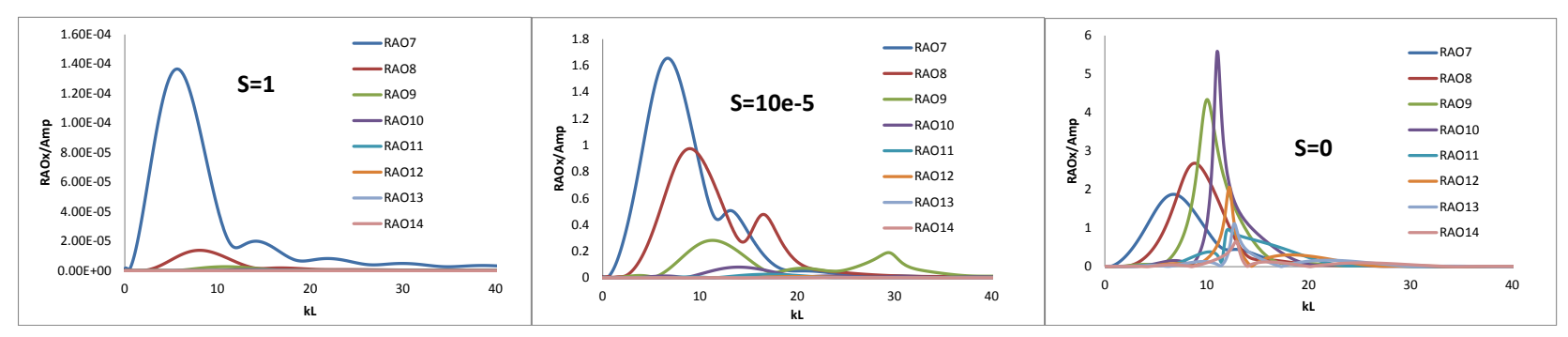

Figure 8. Nondimensional RAOs of elastic slender barge with stiffness factor $S=1, S=10 e-5, S=0$ for the first 8 deformable modes
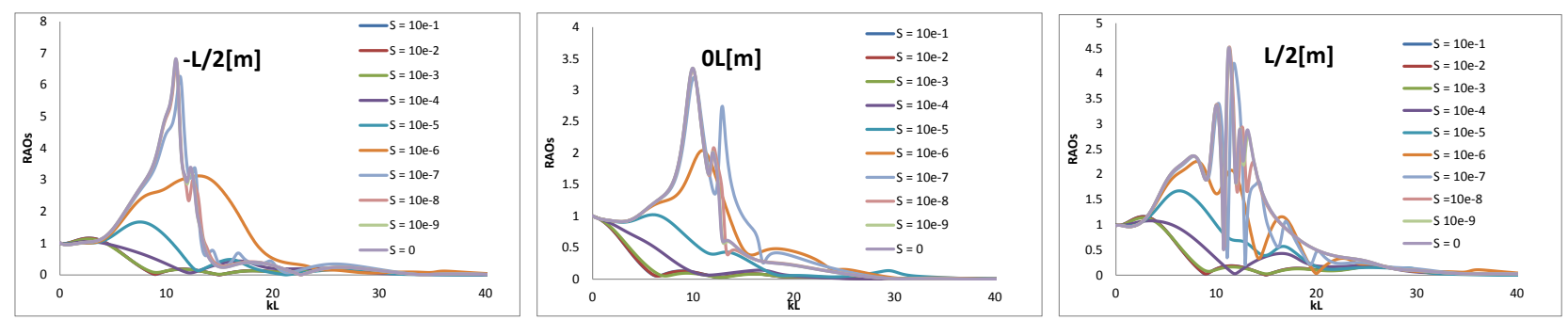

Figure 9. Sum of nondimensional RAOs Eq. (22) for elastic slender barge with a range of nondimensional stiffness factor $S=E I / \rho g L^{5}$ 
Table 1. RAOs and sum of 8 free-free beam loads at L/4 and $\mathrm{L} / 2$ (bow) at the frequency with wavelength equals floating body length

\begin{tabular}{ccccc}
\hline Mode & L/4 & L/4-Sum & L/2 & L/2-Sum \\
\hline $\mathbf{7}$ & 0.077235 & 0.077235 & 0.778619 & 0.778619 \\
$\mathbf{8}$ & 0.062694 & 0.139929 & 0.107215 & 0.885834 \\
$\mathbf{9}$ & 0.003279 & 0.143209 & 0.005280 & 0.891114 \\
$\mathbf{1 0}$ & 0.000484 & 0.143693 & 0.001891 & 0.893005 \\
$\mathbf{1 1}$ & 0.000032 & 0.143725 & 0.000115 & 0.893120 \\
$\mathbf{1 2}$ & 0.000170 & 0.143894 & 0.000258 & 0.893378 \\
$\mathbf{1 3}$ & 0.000002 & 0.143897 & 0.000003 & 0.893382 \\
$\mathbf{1 4}$ & 0.000015 & 0.143912 & 0.000057 & 0.893439 \\
\hline
\end{tabular}

The derivative of the shear force is used to obtain the load distribution Eq. (23) ${ }^{[5]}$

$L(q)=\frac{d V}{d q}=\sum_{j=1}^{K} F_{j} \cdot u_{j}^{\prime \prime \prime \prime}(q)$

where $F_{j}$ is the unknown force coefficients. The first and the second integrations of Eq. (23) are used to determine the shear force and bending moment respectively. The equation of motion in Eq. (1) is used to determine the unknown force coefficient $F_{j}$ in time domain with Eq. (24).

$F_{j}(t)=k_{j k} x_{k}(t)$

$=\int_{-\infty}^{\infty} d \tau K_{j D}(t-\tau) \zeta(\tau)$

$-\sum_{j=1}^{K}\left\{\left(M_{j k}+a_{j k}\right) \ddot{x}_{k}(t)+b_{j k} \dot{x}_{k}(t)+\left(C_{j k}+c_{j k}\right) x_{k}(t)\right.$

$\left.+\int_{0}^{t} d \tau K_{j k}(t-\tau) \dot{x}_{k}(\tau)\right\}$ and in the frequency domain with Eq. (25)

$F_{j}(\omega)=k_{j k} \xi_{k}(\omega)=X_{j}(\omega)-\sum_{j=1}^{K}\left\{-\omega^{2}\left(M_{j k}+A_{j k}(\omega)\right)\right.$

$\left.+i \omega B_{j k}(\omega)+C_{j k}\right\} \xi_{k}(\omega)$

Fig. 10 shows the shear forces as a function nondimensional wave number at symmetric locations in longitudinal direction of the elastic slender barge.

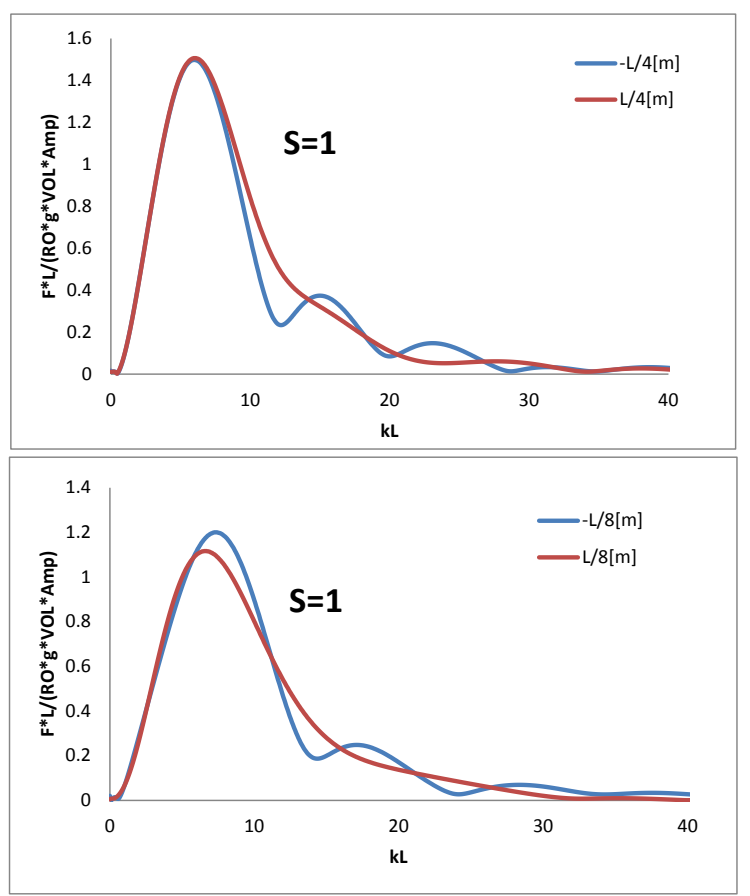

Figure 10. Nondimensional shear force of elastic slender barge Eq. (23) with stiffness factor $\mathrm{S}=1$ (completely rigid) and (-) is for aft of mid-ship, (+) for front of mid-ship

The shear force and bending moment are numerically tested to determine the effects of the hydroelasticity in Fig. 11 and 12 at which nondimensional shear force and bending moment are presented at the location of $\mathrm{q}=-\mathrm{L} / 4$, $\mathrm{q}=0$, and $\mathrm{q}=\mathrm{L} / 4$ in arrange of stiffness factor $\mathrm{S}$.

It can be observed from Fig. 11 that the bending moment and shear force decays to zero when the nondimensional stiffness factor approach the fully flexible condition

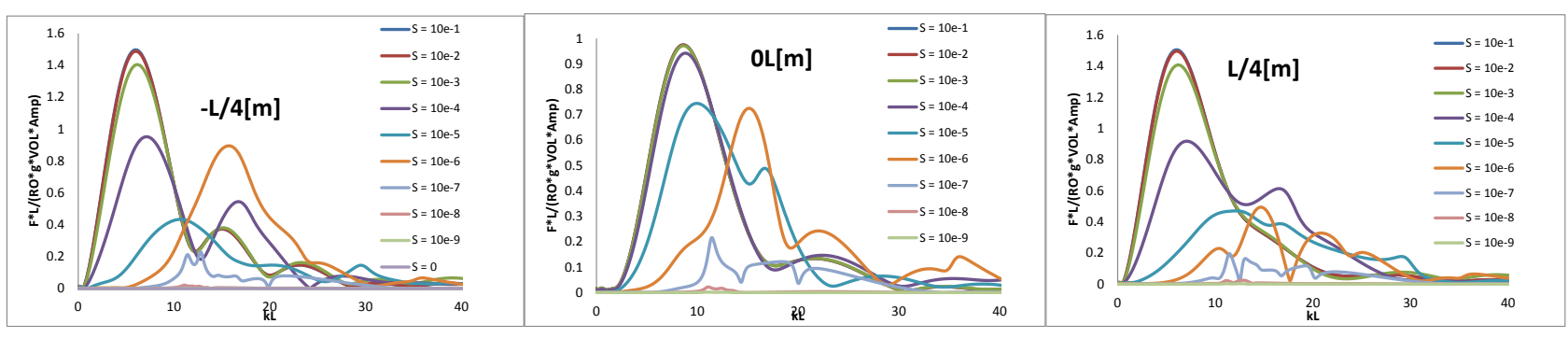

Figure 11. Nondimensional shear forces of elastic slender barge Eq. (23) with a range of non-dimensional stiffness factor $S=E I / \rho g L^{5}$ 

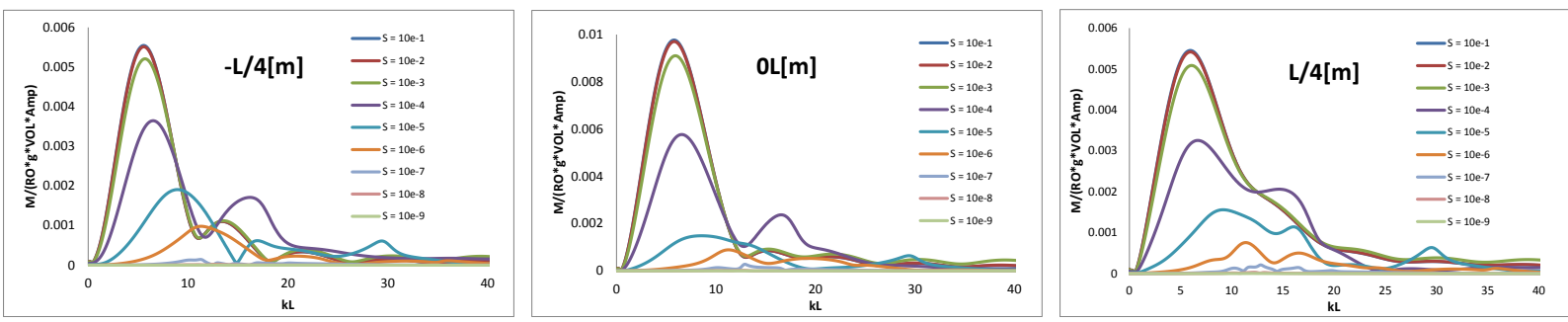

Figure 12. Nondimensional bending moments of elastic slender barge Eq. (23) in a range of nondimensional stiffness factor $S=E I / \rho g L^{5}$

of $\mathrm{S}=0$. The effects of the hydroelasticity for elastic slender barge play significant role at $10^{-8}<\mathrm{S}<10^{-2}$.

\subsubsection{Validation of ITU-WAVE Numerical Results with Experimental Results of a Flexible Barge}

The experimental results ${ }^{[23]}$ for vertical RAOs (deflection) at head seas and zero forward speed are used to validate the in-house ITU-WAVE computational numerical results of the elastic barge in Fig. 1. The length L, length to draught ratio $\mathrm{L} / \mathrm{T}$, and length to beam ratio $\mathrm{L} / \mathrm{B}$, vertical bending stiffness EI of the elastic flexible barge are given with $2.445 \mathrm{~m}, 20.375,4.075$, and $175 \mathrm{Nm}^{2}$ respectively. The given length dimension and vertical bending stiffness results in the prediction of nondimensional stiffness factor $S=\frac{E I}{\rho g L^{5}}=1.99 \times 10^{-4}$ implying that hydroelastic effect is expected to be significant. Total 1080 elements are used to discretise the flexible barge with 5 elements in vertical direction, 10 elements in transverse direction, 49 elements in longitudinal direction whilst $t \sqrt{g / L}=0.05$ is used for nondimensional time step size for ITU-WAVE numerical prediction. It can be seen from Fig. 13 that ITU-WAVE numerical results show acceptable level of agreement with the experimental results ${ }^{[23]}$ for vertical deflection (RAOs).

\subsubsection{Validation of ITU-WAVE Numerical Tool with Experimental Results of a Stiff Wigley Hull Form}

ITU-WAVE numerical results of heave and pitch RAOs, and bending moment and shear force are validated with experimental results ${ }^{[22]}$ of Wigley hull form in Fig. 14 with $\mathrm{Fn}=0.2$, heading angle $\beta=180^{\circ}$, length $\mathrm{L}=2.5 \mathrm{~m}$, length to draught ratio $\mathrm{L} / \mathrm{T}=18$, length to beam ratio $\mathrm{L} /$ $\mathrm{B}=7$.

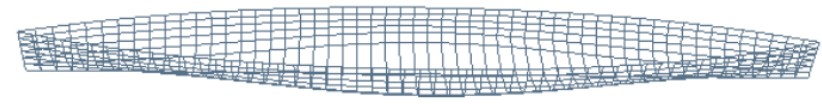

Figure 14. Total 648 panels is used to discretise Wigley form with 12 panels along the girth direction and 54 panels in longitudinal direction

The convergence test results are presented in Fig. 15 for heave and pitch IRFs. The convergence test is done both with respect to nondimensional time step $(t \sqrt{g / L}$ $=0.01,0.03,0.05)$ and panel numbers $(64,144,225,324$, 441). The nondimensional time step $t \sqrt{g / L}=0.05$ and panel number 324 on half part of Wigley hull form due to symmetry are used for the numerical predictions of ITU-WAVE numerical results as the numerical results are
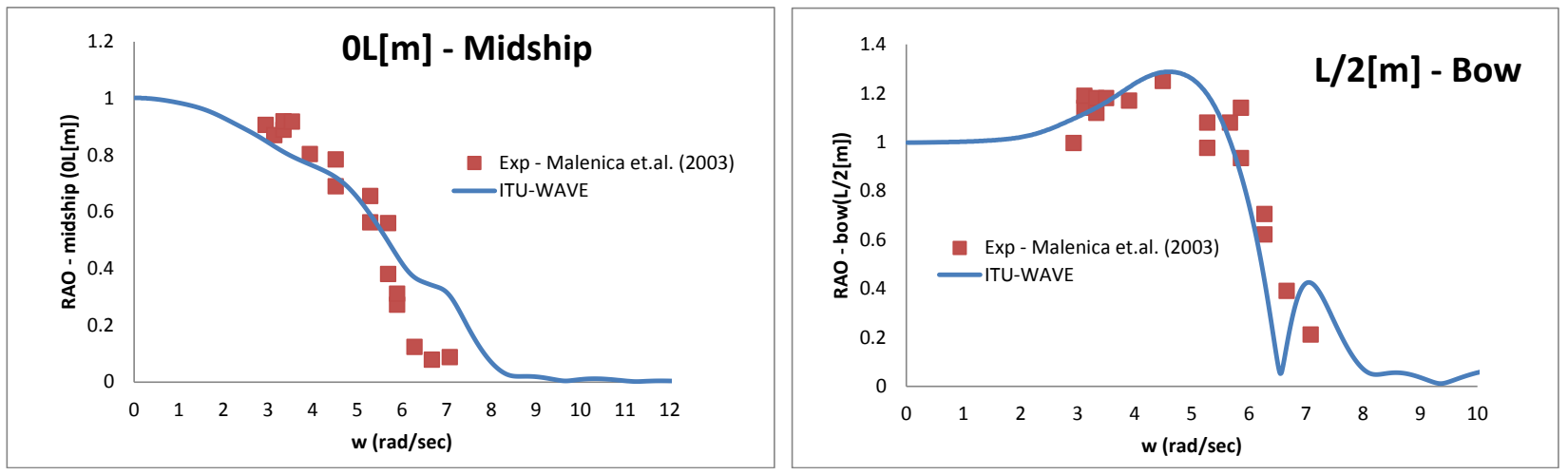

Figure 13. Vertical RAOs of flexible barge at mid-ship ( 0 ) and bow $(\mathrm{L} / 2)$ with heading angle $\beta=180^{\circ}, \mathrm{Fn}=0.0$ and stiffness factor $S=E I / \rho g L^{5}=1.99 \times 10^{-4}$ 
converged at these nondimensional time step and panel number.

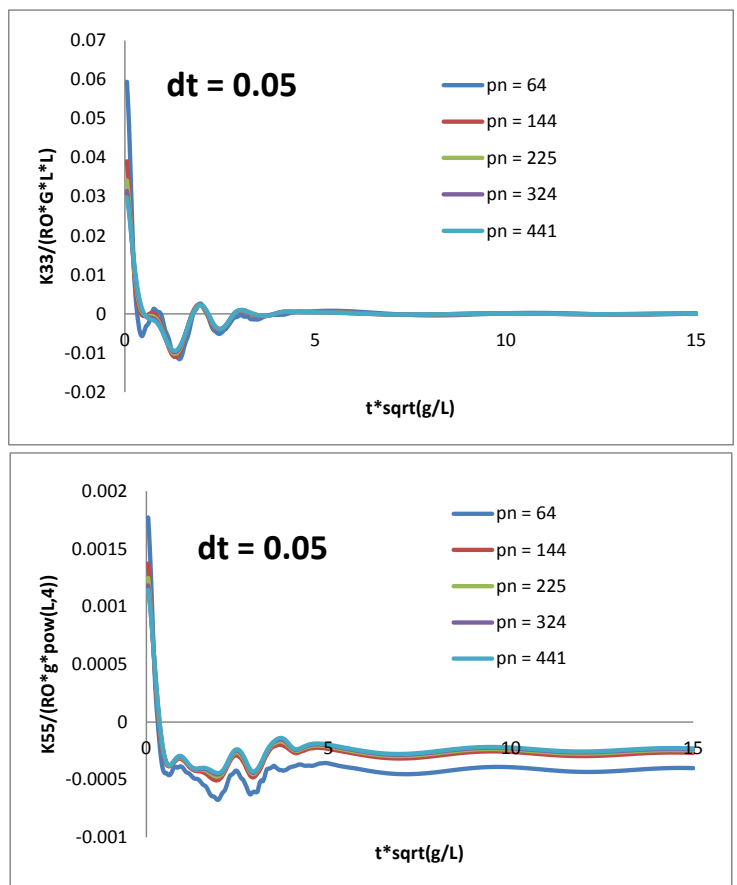

Figure 15. Nondimensional radiation heave and pitch IRFs of Wigley hull form at $\mathrm{Fn}=0.2$

The convergence test is presented in Fig, 16 for exciting heave and pitch IRFs and the numerical results of ITU-WAVE are converged with nondimensional time step $t \sqrt{g / L}=0.05$ and panel number 324 on half part of Wigley hull form due to symmetry as in radiation convergence test.

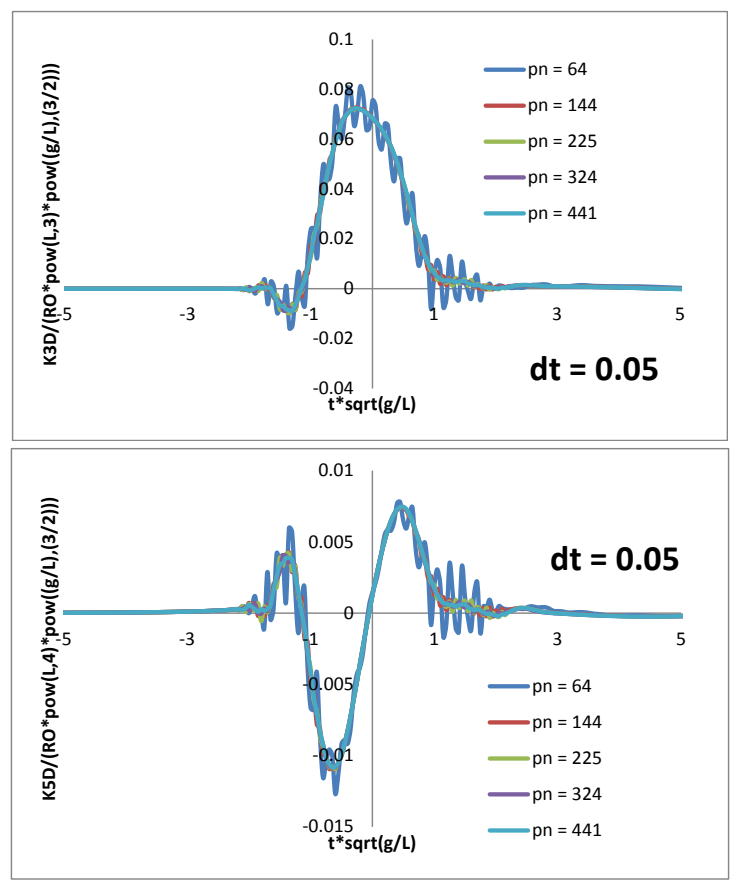

Figure 16. Nondimensional exciting heave and pitch IRFs of Wigley hull form at $\mathrm{Fn}=0.2$ and $\beta=180^{\circ}$
The heave and pitch RAOs of ITU-WAVE numerical results at heading angle $\beta=180^{\circ}$ are presented in Fig. 17 together with experimental results ${ }^{[22]}$ for comparison purpose which show satisfactory agreement. The numerical solution of the RAOs in Fig. 17 are obtained using the frequency domain coupled heave-pitch equation of motion ${ }^{[28]}$. The frequency domain radiation and exciting parameters for frequency domain equation motion are obtained with Fourier transform of IRFs of Fig. 15 and Fig. 16 for radiation and exciting IRFs respectively.
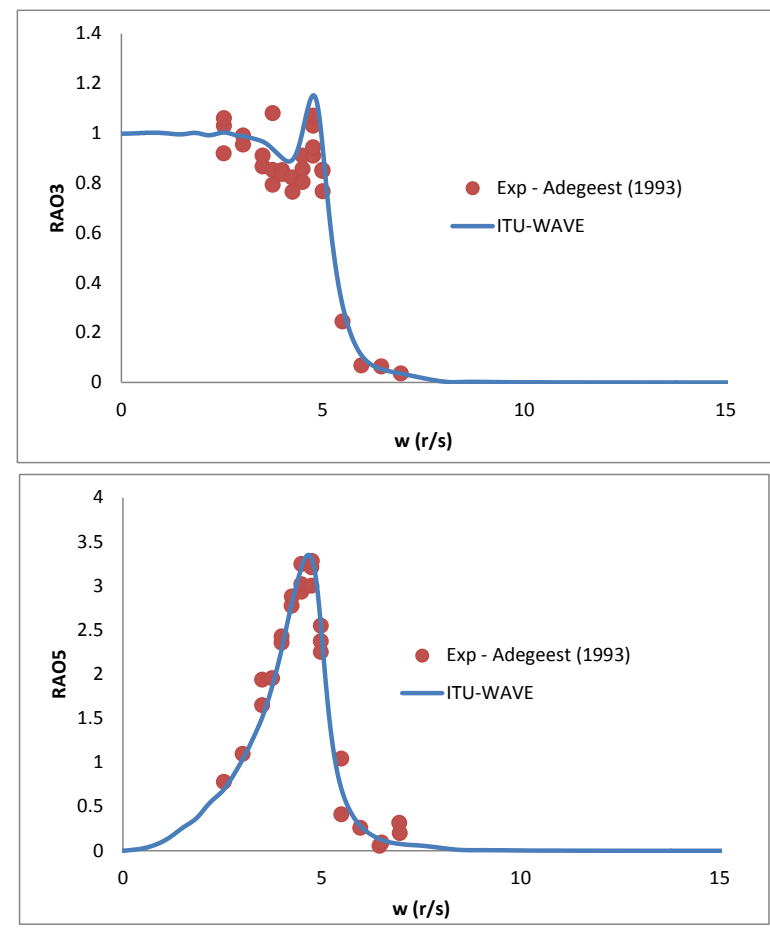

Figure 17. Heave and pitch RAOs of Wigley hull form at

$$
\mathrm{Fn}=0.2 \text { and } \beta=180^{\circ}
$$

If the floating body does not deform much compared to rigid body motion, it can be considered as stiff structure implying that contribution of rigid body modes to the disturbance of the pressure field is much greater compared o elastic deformable modes. In this case Eq. (24) and Eq. (25) can be modified to take only contribution of rigid body modes for unknown force coefficients as in presented in Eq. (24a) and Eq. (25a) in which the summation boundary 2 is used for heave and pitch rigid body modes.

$$
\begin{aligned}
& F_{j}(t)=k_{j k} x_{k}(t) \\
& =\int_{-\infty}^{\infty} d \tau K_{j D}(t-\tau) \zeta(\tau) \\
& -\sum_{k=1}^{2}\left\{\left(M_{j k}+a_{j k}\right) \ddot{x}_{k}(t)+b_{j k} \dot{x}_{k}(t)+\left(C_{j k}+c_{j k}\right) x_{k}(t)\right.
\end{aligned}
$$


$\left.+\int_{0}^{t} d \tau K_{j k}(t-\tau) \dot{x}_{k}(\tau)\right\}$

$F_{j}(\omega)=k_{j k} \xi_{k}(\omega)=X_{j}(\omega)-\sum_{k=1}^{2}\left\{-\omega^{2}\left(M_{j k}+A_{j k}(\omega)\right)\right.$

$\left.+i \omega B_{j k}(\omega)+C_{j k}\right\} \xi_{k}(\omega)$

The shear force and bending moment of Wigley hull form at heading angle $\beta=180^{\circ}$ and Froude number $F n=0.2$ are presented in Fig. 18 in which the present ITUWAVE computational results are compared with the experimental results ${ }^{[22]}$. Eq. (25a) is used to obtain the force coefficients $F_{j}(\omega)$ which is then used for the prediction of the shear force and bending moment in Eq. (23).

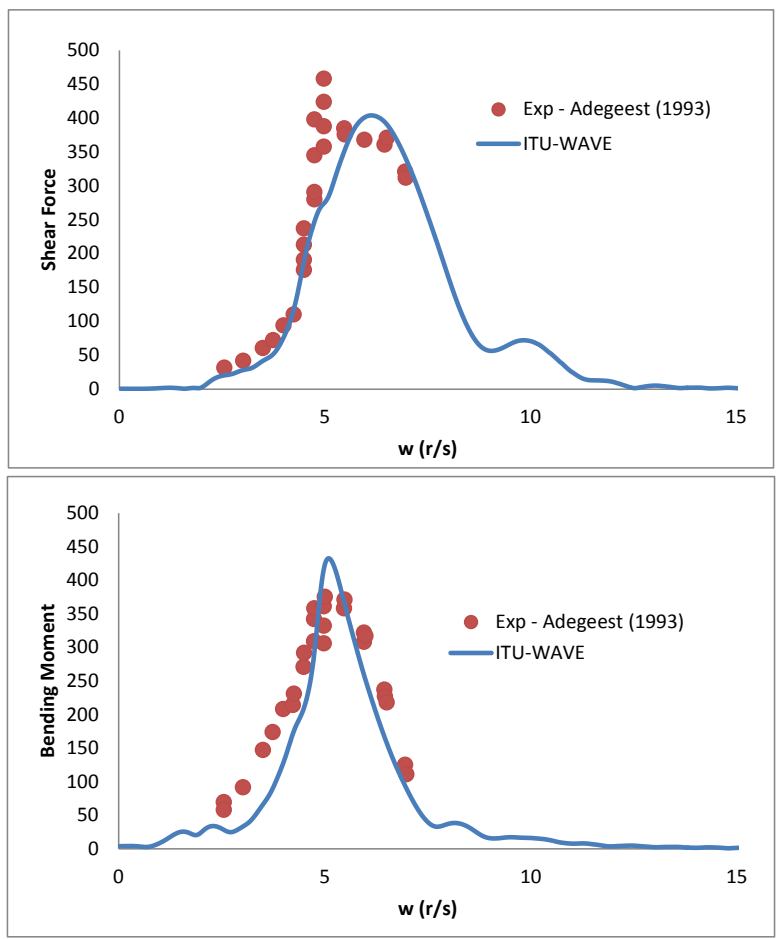

Figure 18. Shear force and bending moment of Wigley hull form at Froude number $\mathrm{Fn}=0.2$ and heading angle $\beta=180^{\circ}$ using 8 free-free Euler-Bernoulli elastic beam modes

\section{Conclusions}

The application of ITU-WAVE computational tool is extended to include the hydroelastic behaviour of the floating marine structures in the present paper. The hydrodynamic part is solved with a three-dimensional potential panel method whilst Euler Bernoulli beam analysis in which modeshap is defined analtically is used for the prediction of the structural behaviour.

A non-dimensional structural stiffness parameter
$S=E I / \rho g L^{5}$ is used and depending on this stiffness parameter the hydroelastic effects of floating slender barge are studied for RAOs, bending moments, and shear forces. It is found out that the hydroelastic effects are dominant in the range of $10^{-8}<\mathrm{S}<10^{-2}$ for a slender barge. A Wigley hull form is then studied as a stiff structure in order to determine the effects of elastic modes due to rigid body modes only which are coupled with elastic modes. As opposite to the traditional approaches, the effects of the different incident wave lengths and geometry of floating bodies are taken into account for the prediction of bending moments and shear forces in the present study.

Numerical results (including the added-mass and damping coefficients as well as the amplitudes of exciting forces and phase angles which can be obtained by Fourier transform of radiation and exciting IRFs respectively) were presented to demonstrate the convergence of the developed computer code for the radiation and exciting IRFs. The numerical predictions for heave and pitch RAOs, bending moment, and shear force at mid-ship of Wigley hull form are shown to be in satisfactory agreement with the experimental results.

\section{References}

[1] Bishop, R.E.D, Price, W.G. Hydroelasticity of Ships. Cambridge University Press, 1979.

[2] Kara, F. Time Domain Hydrodynamics and Hydroelastic Analysis of Floating Bodies with Forward Speed. Ph.D. Thesis, University of Strathclyde in Glasgow, Scotland, UK, 2000.

[3] Kara, F., Vassalos, D. Hydroelastic Analysis of Cantilever Plate in Time Domain. Ocean Engineering, 2007, 34, 122-132.

[4] Newman, J. N. Wave effect on deformable bodies. Applied Ocean Research, 1994, 16, 47-59.

[5] Kara, F. Time Domain Predictions of Hydroelasticity of Floating Bodies. Applied Ocean Research, 2015, $51,1-13$.

[6] Gran, S. A Course in Ocean Engineering. Elsevier, Amsterdam, 1992.

[7] Korvin-Kroukovsky, B.V., Jacobs, W.R. Pitching and Heaving Motions of a Ship in Regular Waves. Transaction of the Society of Naval Architects and Marine Engineers, 1957, 65, 590-632.

[8] Salvensen, N., Tuck, E.O., Faltinsen, O. Ship Motions and Sea Loads Transactions of the Society of Naval Architects and Marine Engineers, 1970, 78, 250-287.

[9] Liapis, S. Time Domain Analysis of Ship Motions. Report No. 302, The Department of Naval Architecture and Marine Engineering, The University of 
Michigan, Ann Arbor, Michigan, USA, 1985.

[10] King, B.W. Time Domain Analysis of Wave Exciting Forces on Ships and Bodies. Report No. 306, The Department of Naval Architecture and Marine Engineering, The University of Michigan, Ann Arbor, Michigan, USA, 1987.

[11] Nakos, D., Sclavounos, P.D. Ship Motions by a Three Dimensional Rankine Panel Method. Proceedings of the 18th Symposium on Naval Hydrodynamics, Ann Arbor, Michigan, 1990:21-41.

[12] Kim, K-H., Bang, J-S., Kim, J-H., Kim, Y., Kim, S-J., Kim Y. Fully coupled BEM-FEM analysis for ship hydroelasticity in waves. Marine Structures, 2013, 33, 71-99.

[13] Xia, J., Wang, Z., Jensen, J.J. Nonlinear Wave Loads and Ship Responses by a Time Domain Strip Theory. Marine Structures, 1998, 11(3):101-123.

[14] Newman, J.N. The Theory of Ship Motions. Advances in Applied Mechanics, 1978, 18, 221-283.

[15] Kara, F. Multibody Interactions of Floating Bodies with Time Domains Predictions. Journal of Waterway, Port, Coastal and Ocean Engineering, 2020, 146 (5).

[16] Kara, F. Time Domain Prediction of Seakeeping Behaviour of Catamarans. International Shipbuilding Progress, 2016, 62 (3-4):161-187.

[17] Kara, F. Time Domain Prediction of Power Absorption from Ocean Waves with Wave Energy Converters Arrays. Renewable Energy, 2016, 92, 30-46.

[18] Kara, F. Time Domain Prediction of Added Resistance of Ships. Journal of Ship Research, 2011, 55(3):163-184.

[19] Kara, F. Time Domain Prediction of Power Absorption from Ocean Waves with Latching Control. Renewable Energy, 2010, 35, 423-434.
[20] Kara, F., Vassalos, D. Time Domain Computation of Wavemaking Resistance of Ships. Journal of Ship Research, 2005, 49 (2):144-158.

[21] Kara, F., Vassalos, D. Time Domain Prediction of Steady and Unsteady Marine Hydrodynamic Problem. International Shipbuilding Progress, 2003, 50(4):317-332.

[22] Adegeest, L. Experimental investigation of the influence of bow flare and forward speed on the nonlinear vertical motions, bending moments and shear forces in extreme regular wave conditions. Laboratory of Ship Hydromechanics, Delft University of Technology, Report Nr. 993, The Netherlands, 1994.

[23] Malenica, S., Molin, B., Remy, F., Senjanovic, I. Hydroelastic response of a barge to impulsive and non-impulsive wave loads. Hydroelasticity in Marine Technology, Oxford, UK, 2003:107-115.

[24] Cummins, W.E. The Impulse Response Function and Ship Motions. Shiffstechnik, 1962, 9, 101-109.

[25] Newman, J. N. Efficient hydrodynamic analysis of very large floating structures. Marine Structures, 2005, 18, 169-180.

[26] Ogilvie, T.F. Recent Progress Toward the Understanding and Prediction of Ship Motions. Proceedings of the 5th Symposium on Naval Hydrodynamics, Office of Naval Research, Washington, D.C., 1964:3-128.

[27] Bishop, R.E.D., Price, W.G., Y. Wu. A General Linear Hydroelasticity Theory of Floating Structures Moving in a Seaway. Philosophical Transactions of the Royal Society of London, Series A, 1986, 316, 375-426.

[28] Lewis, E. V. Principles of Naval Architecture: volume III motions in waves and controllability, second revision, 1989. 